\title{
The Instability of an Electrohydrodynamic Viscous Liquid Micro-Cylinder Buried in a Porous Medium: Effect of Thermosolutal Marangoni Convection
}

\author{
Galal M. Moatimid and Mohamed A. Hassan \\ Department of Mathematics, Faculty of Education, Ain Shams University, Roxy, Cairo 11511, Egypt \\ Correspondence should be addressed to Mohamed A. Hassan; m_a_hassan_gk@hotmail.com
}

Received 9 November 2012; Revised 4 June 2013; Accepted 5 June 2013

Academic Editor: Zhijun Zhang

Copyright @ 2013 G. M. Moatimid and M. A. Hassan. This is an open access article distributed under the Creative Commons Attribution License, which permits unrestricted use, distribution, and reproduction in any medium, provided the original work is properly cited.

\begin{abstract}
The electrohydrodynamic (EHD) thermosolutal Marangoni convection of viscous liquid, in the presence of an axial electric field through a micro cylindrical porous flow, is considered. It is assumed that the surface tension varies linearly with both temperature and concentration. The instability of the interface is investigated for the free surface of the fluid. The expression of the free surface function is derived taking into account the independence of the surface tension of the heat and mass transfer. The transcendental dispersion relation is obtained considering the dependence of the surface tension on the heat and mass transfer. Numerical estimations for the roots of the transcendental dispersion relation are obtained indicating the relation between the disturbance growth rate and the variation of the wave number. It is found that increasing both the temperature and concentration at the axial microcylinder has a destabilizing effect on the interface, according to the reduction of the surface tension. The existence of the porous structure restricts the flow and hence has a stabilizing effect. Also, the axial electric field has a stabilizing effect. Some of previous analytical and experimental results are recovered upon appropriate data choices.
\end{abstract}

\section{Introduction}

The fluid flow in microjet has various applications in medical, biomedical, computer chips and chemical separations. Advent of microelectrical mechanical systems is one of the major advances in industrial technologies. Also, microjets are fundamental to connecting different devices, utilized in biochemical reaction chamber, in physical particle separation, in inkjet print heads, in infrared detectors, in diode lasers, in miniature gas chromatographs, and in heat exchanger for cooling computer chips. Understanding the flow characteristics through a microjet is very important in determining the free surface instability of the fluid flow. The augmentation of heat transfer from a solid cylinder wrapped with a porous layer was considered by Bhattacharyya and Singh [1]. An experimental analysis of unsteady heat and moisture transfer around a heated cylinder buried into a porous medium was performed by Moya et al. [2]. Furlani [3] studied the temporal instability of an infinite Newtonian cylindrical microjet (as a cylindrical fluid surface) that is subjected to a sinusoidal variation of surface tension along its length. Furlani and Hanchak [4] developed the same problem to study numerically the nonlinear analysis of the deformation and breakup of viscous microjets. The instability of non-Newtonian liquid jets (viscoelastic and power law) was investigated by Gao [5] and Gao and Ng [6]. The dispersion relation between the growth rate and the wave number for a non-Newtonian cylindrical liquid jets was derived and the instability behavior of viscoelastic jets was investigated.

The interaction of electric fields with moving fluids makes EHD a very complicated phenomenon. The process of EHD is dependent on many parameters and properties of the liquid and environment of the flow. On the other hand, EHD can be considered as the branch of fluid mechanics concerned with the electrical force effects or as the part that is involved in the influence of moving media on electric fields. It is thus concerned with the interaction between electrical fields and free or polarized charges in fluids. In the EHD stability, the 
electric field plays an important role in many practical problems of chemical engineering, biophysics and many other fields. Mohamed and Nayyar [7] have investigated the stability of a cylindrical jet of incompressible inviscid liquid in the presence of an axial electric field. They showed that the uniform axial electric field has a strong stabilizing influence on the cylindrical interface for short and long wavelengths in all symmetric and asymmetric modes of perturbation.

Marangoni boundary layers are dissipative layers which may occur along liquid-liquid or liquid-gas interfaces. When a free liquid surface is present, the surface tension variation is resulting from the temperature gradients along the surface. It can also induce a motion within the fluid which is called thermocapillary flow (thermal Marangoni convection) [8]. The surface tension gradients that are responsible for Marangoni convection depend on both temperature and/or concentration gradients. Earliest work in this field was first investigated by Napolitano $[9,10]$. Surfactants or surface contaminants are used to control the stability of the free surface by lowering surface tension. Variations in surface tension that are introduced by surfactant can substantially alter interfacial evolution and flow. The effect of surfactants on liquid has been studied by many authors [11-15]. McGough and Basaran [16] studied the breakup mechanisms of the fluid threads containing surfactant by solving the Navier-Stokes equations. The effect of the Prandtl number on Marangoni convection over a flat plate was studied by Christopher and Wang [17]. Physically, the heat and mass transfer across the interface play an essential role in determining the flow field in some cases. For instance, when the fluid is boiling, whether it is a film or bubble, the motion of the film and bubbles is mainly dependent on the effect of mass and heat transfer. In chemical industry, the interfacial stability problems are important in the presence of mass and heat transfer. The mechanism of heat and mass transfer across an interface is of a great importance in numerous industrial and environmental problem processes. These include the design of many types of contacting equipment, for example, boilers, condensers, evaporators, gas absorbers, pipelines, chemical reactors, and nuclear reactors. The effect of heat and mass transfer in the absence of gravity on the surface tension was studied by Straub [18].

The flow through porous media is usually described by the Darcy's law that relates the movement of the fluid to the pressure gradient acting on a parcel of the fluid. Meanwhile, the Darcy's equations are not the fundamental equations for the flow through porous media. It is an approximation for the balance of linear momentum for the fluid flow through a porous solid within the context of mixtures. For example, the interactive force between the fluid and the porous medium, the frictional effects due to viscosity, and the inertial nonlinearities are not included in the Darcy's model [19]. These effects can be included in more generality model like Brinkman-Darcy's model. The basic assumptions that lead to the Brinkman-Darcy equation were illustrated by Rajagopal [19]. Elcoot and Moatimid [20] studied the instability of finitely conducting cylindrical flows through porous media, under the influence of an axial electrostatic field. They found that, under certain conditions, the field may have a stabilizing or destabilizing effect. An experimental study of the steam injection into a porous media and the stability of the interface between the steam and the water was carried out by Catton and Chung [21].

The aim of this work is to discuss the effect of axial electric field with the existence of the porous structure on the stability of the microcylindrical flow of a viscous liquid past a microsolid cylindrical surface. Also, the effect of the Marangoni thermosolutal convection (according to the dependence of the surface tension on the heat and mass transfer) is discussed. The main construction of the problem is as follows. Firstly, we studied the problem according to spatial surface tension. So, the expression of the free surface function is obtained. Secondly, the problem is studied in the presence of the heat and mass transfer rates with thermosolutal surface tension. The dispersion relation, in the latter case, is obtained as a transcendental function of the growth rate and the wave number. Finally, we studied the effect of the porous medium and the axial electric field on the stability of viscous liquid interface and tabulated results are obtained.

To clarify the problem, in Section 2, the physical description of the problem including the basic equations that govern the motion is presented. Section 3 is devoted to introducing the interfacial conditions for both the fluid and the electric field together with the boundary conditions. The method of solution according to the normal modes technique is presented in Section 4. In Section 5, the instability analysis in the absence of the heat and mass transfer is studied and the expression of the free surface function is derived. The transcendental dispersion relation in the presence of the heat and mass transfer is obtained in Section 6. Throughout Section 7, we introduced numerical estimations and gave a discussion for the stability picture according to the relation between the growth rate and the wave number. Finally, in Section 8 , we give concluding remarks for this study based on the obtained results of the stability analysis.

\section{Formulation of the Problem}

Consider a microcylindrical layer of viscous fluid with density $\rho$, viscosity $\mu$, and radius $r_{0}$ flowing past an axial solid cylinder of infinitely small radius. The flow is surrounded by a vacuum (which has zero density and zero viscosity) with hypothetical sufficiently large radius $R_{2}$. The axial microsolid cylinder is elongated along the $z$-axis. It is earthed and has a radius $R_{1}$ (where $R_{1} \ll R_{2}$ ). The axial microcylindrical solid rod, which induces temperature and concentration gradients, is considered to be at a constant temperature $T_{1}$ and constant concentration $C_{1}$. The subscripts (1) and (2) refer to the liquid and the surrounding vacuum, respectively. The fluid and the vacuum are influenced by an external uniform electric field of intensity $E_{0}$ which acts along the positive $z$-axis. The dielectric constants for the liquid and the vacuum are $\varepsilon_{1}$ and $\varepsilon_{2}$, respectively. The interface between the fluid and the surrounding vacuum is considered to be free of charges. The flow jet is considered as a microcylindrical porous structure with Darcy's coefficient $v$, while its porosity is assumed to be unity (this will be explained physically later). Also, we neglect the effects of gravity. Considering the previous hypotheses, the model may be illustrated graphically as in Figure 1. 


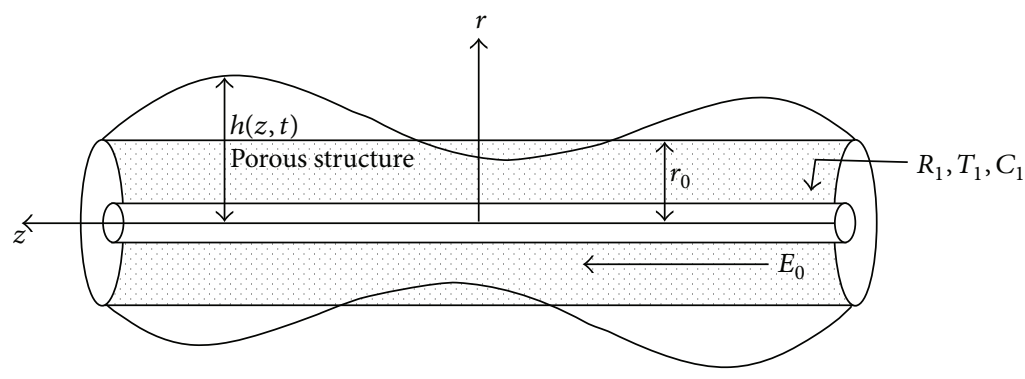

Figure 1: Physical model and flow cylindrical geometry.

After making a small disturbance, the interface function becomes

$$
F(r, z, t)=r-h(z, t),
$$

and the unit outward normal vector $\widehat{n}$ and the unit tangential one $\hat{t}$ are given by

$$
\begin{aligned}
& \widehat{n}=\frac{1}{\sqrt{1+h_{z}^{2}}} \widehat{r}-\frac{h_{z}}{\sqrt{1+h_{z}^{2}}} \widehat{z}, \\
& \widehat{t}=\frac{h_{z}}{\sqrt{1+h_{z}^{2}}} \widehat{r}+\frac{1}{\sqrt{1+h_{z}^{2}}} \widehat{z},
\end{aligned}
$$

where $\widehat{r}$ and $\widehat{z}$ are the unit vectors along the radial and axial cylindrical coordinate directions and $h_{z}=\partial h / \partial z$.

According to the assumption of the viscosity of the fluid, considering the existence of the porous structure and the heat and mass transfer, the basic equations of the problem may take the following form $[3,19]$.

The continuity equation (incompressibility condition) requires

$$
\nabla \cdot \underline{v}=0
$$

The balance of linear momentum (Navier-Stokes) gives

$$
\rho\left[\frac{1}{\zeta} \frac{\partial \underline{v}}{\partial t}+\frac{1}{\zeta^{2}}(\underline{v} \cdot \underline{\nabla}) \underline{v}\right]=-\nabla P+\mu_{\mathrm{eff}} \nabla^{2} \underline{v}-\nu \underline{v} .
$$

The balance of energy is

$$
\frac{D T}{D t}=\alpha \nabla^{2} T
$$

And finally the concentration equation becomes

$$
\frac{D C}{D t}=\beta \nabla^{2} C
$$

In the previous equations (3)-(6), $\zeta$ is the porosity of the porous medium, $\mu_{\text {eff }}$ is the effective viscosity of the fluid ( $\mu_{\text {eff }}=\mu / \zeta$ and if $\zeta=1$ then $\mu_{\text {eff }}=\mu$ as we mention in the next paragraph), and $(D / D t)=(\partial / \partial t)+(\underline{v} \cdot \underline{\nabla}) \underline{v}$. Also, $\alpha$ is the thermal diffusivity, $\beta$ is the mass diffusivity, $T$ is the temperature, $C$ is the concentration, $P$ is the pressure, $\underline{v}$ is the velocity vector, $v$ is the Darcy's coefficient, and $\rho$ is the density.
The basic assumptions that lead to the Brinkman-Darcy equation and the form of the basic assumptions that lead to the Brinkman-Darcy equation were illustrated by Rajagopal [19], and can be summarized in the following points.

(1) The porous medium is solid and thus the balance of linear momentum of the porous medium can be ignored.

(2) The interactive force between the fluid and the porous medium is due to the frictional forces only and this force is proportional to the flow velocity which is represented by the term $\nu \underline{v}$, where $\nu=\mu / \lambda$, $\mu$ is the fluid viscosity and $\lambda$ is the permeability of the porous medium.

(3) The frictional effects due to viscosity were taken into account by the term $\mu_{\text {eff }} \nabla^{2} \underline{v}$, where $\mu_{\text {eff }}=\mu / \zeta$.

(4) The flow is unsteady and sufficiently fast, so that the inertial nonlinearities cannot be ignored; thus the term $\left(1 / \zeta^{2}\right)(\underline{v} \cdot \underline{\nabla}) \underline{v}$ needs to be retained.

According to the previous assumptions, the balance of linear momentum can be written as in (4). Also, we want to confirm the following points.

(1) The velocity of the flow through the porous medium is the average of the fluid velocity. This quantity has been given various names by different authors, such as seepage velocity, filtration velocity, superficial velocity, or Darcy velocity [22]. The seepage velocity $\underline{v}$ is related by the velocity of the fluid $\underline{V}$ (where $\underline{V}$ is the flow velocity in the absence of the porous medium) by $\underline{v}=\zeta \underline{V}$.

(2) The porosity $\zeta$ for natural media does not normally exceed 0.6. For beds of solid spheres of uniform diameter $\zeta$ can vary between the limits 0.2595 (rhombohedral packing) and 0.4764 (cubic packing). Nonuniformity of grain size tends to lead to smaller porosities than those for uniform grains, because smaller grains fill the pores formed by larger grains. For man-made materials such as metallic foams $\zeta$ can approach the value 1 (see the tabulated values for the porosity and permeability in [22, page 5]).

(3) The term $\left(1 / \zeta^{2}\right)(\underline{v} \cdot \underline{\nabla}) \underline{v}$ is inappropriate if the flow is sufficiently slow. Then the inertial nonlinearities can be neglected [19]. However, this term needs to 
be retained in the case of highly porous media where the flow is sufficiently fast and at least the irrotational part of this term needs to be retained [22] and for incompressible fluids this term reduces to $\zeta^{-1} \nabla\left[\zeta^{-1} \underline{v} \cdot \underline{v}\right]$, and for highly porous media we can assume $\zeta=1$ (as we assume in the present problem).

(4) Brinkman sets the viscosity $\mu$ and the effective viscosity $\mu_{\text {eff }}$ equal to each other but in general that is not true [22]. The difference between $\mu$ and $\mu_{\mathrm{eff}}$ is due to the momentum dispersion and $\mu_{\mathrm{eff}}$ is dependent on the type of the porous medium as well as the strength of flow, and it is common practice to take $\mu_{\text {eff }}$ equal to $\mu$ for high porosity media [23].

(5) Experimental checks of Brinkman's theory have been indirect and few in number. The Brinkman's equation reduces to Darcy's equation when the spatial length scale is much greater than $\left(\mu_{\text {eff }} \lambda / \mu\right)^{1 / 2}$. Therefore the term $\nabla^{2} \underline{v}$ is negligible in comparison with the term proportional to $\underline{v}$ [22]. But, the Brinkman's model holds only for particles whose size is of order $\vartheta^{3}$, where $\vartheta \ll 1$ is the distance between neighboring particles, and for large particles the fluid filtration is governed by Darcy's law [22].

Because of the quasi-static approximation and in poorly conducting fluid $[24,25]$, the induced magnetic field is negligible. Also, we deal with very small electric conductivity and there is no applied magnetic field. So, the electric field is conservative. Since, the electric field is curl free vector the Maxwell's equations are $\nabla \times \underline{E}=0$ and $\nabla \cdot \varepsilon \underline{E}=0$. So, the electric field has an electric scalar potential $\phi$ such that $\underline{E}=E_{0} \widehat{z}-\underline{\nabla} \phi$. Therefore the electric potential $\phi_{j}$ satisfies the Laplace's equation

$$
\nabla^{2} \phi_{j}=0 \quad(j=1,2) .
$$

The boundary and interfacial conditions for the problem may be displayed in the following section.

\section{Boundary Conditions}

The boundary conditions adopted here must be classified into three categories. The first relates the hydrodynamic part at the boundaries. The second is concerned with the electric part at the boundaries. Meanwhile, the third relates the combination condition of the electric and hydrodynamic balance of the stress tensor components at the interface between the liquid and the surrounding vacuum.

(1) For the hydrodynamic part, because $F$ (in (1)) is a scalar function that is always equal to zero at any point on the fluid interface, its time derivative following any material point on the interface is obviously equal to zero, which means that there is no phase transformation occurring [26, Chapter 2 , page 75$]$. So, the continuity of the normal velocity at the interface requires

$$
\frac{D}{D t}[r-h(z, t)]=0, \quad \text { at } r=h,
$$

where $r$ is the radial distance and $h$ is the radius for the disturbed fluid surface.
According to the microscale of the cylindrical fluid radius, the standard no-slip boundary condition in the classical fluid mechanics does not apply, so that the shear stress at the boundary vanishes (i.e., there is no considerable surface fraction between the fluid and the axial bar) and hence $\partial v_{z} / \partial r=0$ at the axial bar. Meanwhile, the normal velocity must be vanishing at the axial bar. So the boundary conditions for the velocity at the surface of the microcylindrical axis may be written as follows:

$$
v_{r}=0, \quad r=R_{1}, \quad \frac{\partial v_{z}}{\partial r}=0, \quad r=R_{1},
$$

where $R_{1}$ is the microradius of the axial microcylindrical bar such that $R_{1}$ is sufficiently small $\left(R_{1} \ll 1\right)$ and $v_{r}, v_{z}$ are the radial and axial velocity components, respectively.

(2) For the electric part, because there is no surface charges accumulated at the interface, the normal electric displacement must be continuous across the interface. So that $\underline{n} \cdot\|\varepsilon \underline{E}\|=0$ and the interfacial condition for the normal electric field displacement, in the linear form, yields

$$
E_{0} h_{z}\left\|\varepsilon_{j}\right\|+\left\|\varepsilon_{j} \frac{\partial \phi_{j}}{\partial r}\right\|=0, \quad \text { at } r=h,
$$

where the notation \|\| denotes the subtract at the fluid and surrounding vacuum. In other words $\|f\|=f_{1}-f_{2}$. The subscript $j$, in the electric potential $\phi_{j}$ and the dielectric constant $\varepsilon_{j}$, takes the values $j=1,2$ at the fluid and the surrounding vacuum, respectively.

The continuity of the tangential electric filed components at the interface requires $\underline{n} \times\|\underline{E}\|=0$ and in the linear form (where the linear terms only are retained and the nonlinear terms are omitted) becomes

$$
\left\|\frac{\partial \phi_{j}}{\partial z}\right\|=0, \quad \text { at } r=h .
$$

Since the axial microsolid bar and the vacuum at infinity have no electric field, the electric potential at the boundaries satisfies

$$
\underline{\nabla} \phi_{1}=0, \quad r=R_{1}, \quad \underline{\nabla} \phi_{2}=0, \quad r=R_{2} .
$$

(3) The balance of the stress components at the interface may be presented as follows.

At the interface between the liquid and vacuum, the fluid and the electrical stresses must be balanced. The components of these stresses consist of the electric hydrodynamic stresses together with the surface tension force [27]. The combination of the electric stress $\tau_{i j}^{\text {electro }}$ and hydrodynamic stress $\tau_{i j}^{\text {hydro }}$ parts may be presented in the total stress as

$$
\tau_{i j}=\tau_{i j}^{\text {hydro }}+\tau_{i j}^{\text {electro }} .
$$

The electric force density $\underline{F}_{e}$ can be written in terms of the electric field as $\underline{F}_{e}=(\nabla \cdot \varepsilon \underline{E}) \underline{E}$. Since $\nabla \times \underline{E}=\underline{0}$, the electric force can be written as $\underline{F}_{e}=(\nabla \cdot \varepsilon \underline{E}) \underline{E}+(\nabla \times \underline{E}) \times \varepsilon \underline{E}$ and the Maxwell stress tensor $\tau_{i j}^{\text {electro }}$, in the index notation, may be written as [28]

$$
\tau_{i j}^{\text {electro }}=\varepsilon E_{i} E_{j}-\frac{1}{2} \varepsilon E^{2} \Delta_{i j},
$$


meanwhile, the hydrodynamic stress tensor may be described by the constitutive relation

$$
\tau_{i j}^{\text {hydro }}=-P \Delta_{i j}+\mu\left(\frac{\partial v_{i}}{\partial x_{j}}+\frac{\partial v_{j}}{\partial x_{i}}\right)
$$

where $\Delta_{i j}$ is the usual kronecker delta and $v_{i}, v_{j}, x_{i}, x_{j}$ are a general notations for the velocity components and the coordinates, respectively.

According to the simple interface description, which involves only interfacial tension, the forces acting on any segment of an interface are of two kinds. First, there are the bulk pressure and stresses that act on the faces of the interface element and produce a net effect that is proportional to the surface area; second, there is a tensile force that is due to surface or interfacial tension that acts in the plane of the interface at the edges of the surface element and is specified by means of the magnitude of the surface or interfacial tension as a force per unit length [26, Chapter 2, page 76]. So, the stress balance can be written as [3]

$$
\widehat{n} \cdot \tau=-2 H \sigma(z) \widehat{n}+\nabla_{S} \sigma(z),
$$

where $H$ is the curvature of the interface, $\nabla_{S}$ is the surface gradient at the interface, and $\sigma(z)$ is the surface tension.

The previous condition can be decomposed into the normal and tangential stress tensor components as follows.

(i) The normal stress component requires

$$
(\widehat{n} \cdot \tau) \cdot \widehat{n}=-2 H \sigma(z)
$$

(ii) The tangential stress components yield

$$
(\tau \cdot \widehat{n}) \cdot \widehat{t}=\widehat{t} \cdot \nabla_{S} \sigma(z)
$$

where the surface curvature and the gradient at the interface may be given as follows:

$$
\begin{gathered}
H=\frac{1}{2} \nabla \cdot \widehat{n}=\frac{1}{2}\left(\frac{1}{h \sqrt{1+h_{z}^{2}}}-\frac{h_{z z}}{\left(1+h_{z}^{2}\right)^{3 / 2}}\right), \\
\nabla_{S} \equiv \frac{1}{2}(\nabla-\underline{n}(\underline{n} \cdot \nabla))=\widehat{r} \frac{h_{z}}{1+h_{z}^{2}} \frac{\partial}{\partial z}+\widehat{z} \frac{1}{1+h_{z}^{2}} \frac{\partial}{\partial z} .
\end{gathered}
$$

Finally, the normal and tangential stress tensor conditions as given in (17) and (18) (at $r=h$ ) may be written as follows:

$$
\begin{gathered}
-P+\frac{2 \mu}{1+h_{z}^{2}}\left[\frac{\partial v_{r}}{\partial r}+h_{z}^{2} \frac{\partial v_{z}}{\partial z}-h_{z}\left(\frac{\partial v_{r}}{\partial z}+\frac{\partial v_{z}}{\partial r}\right)\right] \\
+\frac{1}{2\left(1+h_{z}^{2}\right)} \| \varepsilon_{j}\left[\left(1-h_{z}^{2}\right)\left(\frac{\partial \phi_{j}}{\partial r}\right)^{2}\right.
\end{gathered}
$$

$$
\begin{aligned}
& -\left(1-h_{z}^{2}\right)\left(E_{0}-\frac{\partial \phi_{j}}{\partial z}\right)^{2} \\
& \left.+4 h_{z} \frac{\partial \phi_{j}}{\partial r}\left(E_{0}-\frac{\partial \phi_{j}}{\partial z}\right)\right] \|
\end{aligned}
$$

$$
=-2 \sigma(z) H
$$

$$
\begin{aligned}
& \frac{\mu}{1+h_{z}^{2}}\left[2 h_{z}\left(\frac{\partial v_{r}}{\partial r}-\frac{\partial v_{z}}{\partial z}\right)\right. \\
&\left.+\left(1-h_{z}^{2}\right)\left(\frac{\partial v_{r}}{\partial z}+\frac{\partial v_{z}}{\partial r}\right)\right] \\
&+\frac{1}{\left(1+h_{z}^{2}\right)} \| \mathcal{E}_{j}\left[h_{z}\left(\frac{\partial \phi_{j}}{\partial r}\right)^{2}-h_{z}\left(E_{0}-\frac{\partial \phi_{j}}{\partial z}\right)^{2}\right. \\
&\left.\quad-\left(1-h_{z}^{2}\right) \frac{\partial \phi_{j}}{\partial r}\left(E_{0}-\frac{\partial \phi_{j}}{\partial z}\right)\right] \|
\end{aligned}
$$

$$
=\widehat{t} \cdot \nabla_{S} \sigma(z) \text {. }
$$

We study the solution of the equations of motion (4) according to the boundary and interfacial conditions in two cases. The first case is obtained in the absence of the heat and mass transfer where the spatial surface tension is independent of the rate of heat and mass transfer. In this case, we follow the same analysis of Furlani [3] to gain the function of the free surface of the microcylindrical fluid flow. In the second one, taking into account the dependence of the surface tension on the heat and mass transfer, the surface tension becomes a function of the time and the axial distance. So, in this case, the dispersion relation may be obtained as a transcendental function and its roots can be computed numerically hence the stability picture may be obtained graphically. Before studying the previous cases, we obtain the solution of the electric field according to the boundary and interfacial conditions.

\section{Solution of the Eclectic Field}

Our analysis will be based on the normal modes technique. Therefore, the electric potential may take the following form:

$$
\phi_{j}(r, z, t)=\varphi_{j}(r) e^{\omega t+i k z}
$$

where $\omega$ is the frequency of the surface wave and $k$ is its wave number which is assumed to be real and positive. Using (7) with the boundary conditions (10)-(12), we may obtain the solution for the electric potential functions as follows:

$$
\begin{aligned}
\phi_{1}(r, z, t) & \\
= & \frac{i r_{0} E_{0}\left(\varepsilon_{2}-\varepsilon_{1}\right) g_{2}(k)}{\varepsilon_{2} g_{1}(k) G_{2}(k)-\varepsilon_{1} g_{2}(k) G_{1}(k)} \\
& \times\left[K_{0}(k r) I_{0}^{\prime}\left(k R_{1}\right)-K_{0}^{\prime}\left(k R_{1}\right) I_{0}(k r)\right] e^{\omega t+i k z},
\end{aligned}
$$




$$
\begin{aligned}
\phi_{2}(r, z, t) & \\
= & \frac{i r_{0} E_{0}\left(\varepsilon_{2}-\varepsilon_{1}\right) g_{1}(k)}{\varepsilon_{2} g_{1}(k) G_{2}(k)-\varepsilon_{1} g_{2}(k) G_{1}(k)} \\
& \times\left[K_{0}(k r) I_{0}^{\prime}\left(k R_{2}\right)-K_{0}^{\prime}\left(k R_{2}\right) I_{0}(k r)\right] e^{\omega t+i k z},
\end{aligned}
$$

where $I_{0}$ and $K_{0}$ are the modified Bessel's functions of first and second kinds of order zero, respectively. The dashes denote the differentiation with respect to $r$ and $i=\sqrt{-1}$. Also, the functions $g_{1}(k), g_{2}(k), G_{1}(k)$, and $G_{2}(k)$ are given in the appendix.

\section{Instability in the Absence of Heat and Mass Transfer}

This section is devoted to solving the equations of motion and the continuity equation (see (3) and (4)) according to the interfacial and boundary conditions (see (8) and (9)) with employing the normal and tangential stress tensor interfacial conditions. We follow the same procedure given by Furlani [3] to get the function that describes the free surface. So, we expand the velocity components and the pressure as a power of $r$ (depending on the microradius of the flow jet), as follows:

$$
\begin{aligned}
& v_{z}(r, z, t)=v_{0}(z, t)+v_{2}(z, t) r^{2}+\cdots, \\
& P(r, z, t)=P_{0}(z, t)+P_{2}(z, t) r^{2}+\cdots .
\end{aligned}
$$

From the continuity equation (3), one gets

$$
v_{r}(r, z, t)=-\frac{\partial v_{0}(z, t)}{\partial z} \frac{r}{2}-\frac{\partial v_{2}(z, t)}{\partial z} \frac{r^{3}}{4}+\cdots
$$

where $v_{0}(z, t), v_{2}(z, t)$ are perturbed velocity functions and $P_{0}(z, t), P_{2}(z, t)$ are the perturbed pressure functions. It is important to notice here that the expansions in (24)-(25) are compatible with the boundary conditions in (9) and with the continuity equation (3). Where $R_{1}$ and $r$ are a microradii of the axial microcylinder and the microporous cylinder that contains the fluid, respectively. So, all terms containing $O(r)$ and $O\left(R_{1}\right)$ or higher orders can be neglected according to their tendency to zero.

According to the previous expansions, the equation of motion (4) for $v_{r}$ can be neglected because it is identical to the lowest order of $r$. Therefore the equation of motion for $v_{z}$ can be written as

$$
\begin{aligned}
\rho\left(\frac{\partial v_{0}}{\partial t}\right. & \left.+v_{0} \frac{\partial v_{0}}{\partial z}\right) \\
& =-\frac{\partial P_{0}}{\partial z}+\mu\left(4 v_{2}+\frac{\partial^{2} v_{0}}{\partial z^{2}}\right)-\nu v_{0}
\end{aligned}
$$

The second order term of the velocity $v_{2}$ can be determined from the tangential stress condition as given in (21); on neglecting the terms of $O(r)$ and all higher orders, it can be written as follows:

$$
\begin{aligned}
v_{2}= & \frac{1}{2 \mu h} \frac{\partial \sigma}{\partial z}+\frac{3 h_{z}}{2 h} \frac{\partial v_{0}}{\partial z}+\frac{1}{4} \frac{\partial^{2} v_{0}}{\partial z^{2}} \\
& +\left\|\frac{\varepsilon_{j} E_{0}}{2 \mu h} \frac{\partial \phi_{j}}{\partial r}-\frac{\varepsilon_{j} E_{0} h_{z}}{\mu h} \frac{\partial \phi_{j}}{\partial z}\right\| .
\end{aligned}
$$

The zero order term of the pressure $P_{0}$ can be determined from the normal stress condition, as given in (20), in the form

$$
P_{0}=-\mu \frac{\partial v_{0}}{\partial z}+2 \sigma H+\left\|\varepsilon_{j} E_{0} \frac{\partial \phi_{j}}{\partial z}\right\| .
$$

Now, substituting from (27) and (28) into (26), we obtain

$$
\begin{aligned}
\rho\left(\frac{\partial v_{0}}{\partial t}\right. & \left.+v_{0} \frac{\partial v_{0}}{\partial z}\right) \\
= & -\frac{\partial}{\partial z}(2 \sigma H) \\
& +\left\|\frac{2 \varepsilon_{j} E_{0}}{h} \frac{\partial \phi_{j}}{\partial r}-\frac{4 \varepsilon_{j} E_{0} h_{z}}{h} \frac{\partial \phi_{j}}{\partial z}-\varepsilon_{j} E_{0} \frac{\partial^{2} \phi_{j}}{\partial z^{2}}\right\| \\
& +\frac{3 \mu}{h^{2}} \frac{\partial}{\partial z}\left(h^{2} \frac{\partial v_{0}}{\partial z}\right)+\frac{2}{h} \frac{\partial \sigma}{\partial z}-\nu v_{0} .
\end{aligned}
$$

The kinematic condition equation (8) gives

$$
\frac{\partial h}{\partial t}=-v_{0} \frac{\partial h}{\partial z}-\frac{h}{2} \frac{\partial v_{0}}{\partial z} .
$$

Also, the axial velocity $v_{0}(z, t)$, the radial position $h(z, t)$, and the surface tension $\sigma(z)$ can be represented in a perturbed and unperturbed parts as follows:

$$
\begin{gathered}
v_{0}(z, t)=u_{0}+u(z, t), \\
h(z, t)=r_{0}(1+\delta(z, t)), \\
\sigma(z)=\sigma_{0}+\sigma_{1}(z),
\end{gathered}
$$

where $u_{0}, r_{0}$, and $\sigma_{0}$ are the constant unperturbed axial streaming velocity, radius, and surface tension, respectively. The analysis may be simplified by using the transformation

$$
\eta=z-u_{0} t
$$

By using (31)-(34) into (29) and (30), after linearizing the resulting equations, we can obtain

$$
\begin{aligned}
& \rho\left(\frac{\partial u}{\partial t}\right)= \frac{1}{r_{0}} \frac{\partial \sigma_{1}}{\partial \eta}+\left\|\frac{2 \varepsilon_{j} E_{0}}{r_{0}} \frac{\partial \phi_{j}}{\partial r}-\varepsilon_{j} E_{0} \frac{\partial^{2} \phi_{j}}{\partial \eta^{2}}\right\| \\
&+ 3 \mu \frac{\partial^{2} u}{\partial \eta^{2}}+\frac{\sigma_{0}}{r_{0}} \frac{\partial \delta}{\partial \eta}+\sigma_{0} r_{0} \frac{\partial^{3} \delta}{\partial \eta^{3}}-\nu u, \\
& \frac{\partial^{2} u}{\partial \eta^{2}}=-2 \frac{\partial^{2} \delta}{\partial \eta \partial t} .
\end{aligned}
$$


Equations (35) can be combined into one equation that determines the free surface function $\delta(\eta, z)$ as follows:

$$
\begin{aligned}
\frac{\partial^{2} \delta}{\partial t^{2}} & +\frac{\partial}{\partial t}\left(\frac{\nu}{\rho} \delta-\frac{3 \mu}{\rho} \frac{\partial^{2} \delta}{\partial \eta^{2}}\right) \\
& +\frac{\sigma_{0}}{2 \rho r_{0}}\left(\frac{\partial^{2} \delta}{\partial \eta^{2}}+r_{0}^{2} \frac{\partial^{4} \delta}{\partial \eta^{4}}\right) \\
& +\left\|\frac{\varepsilon_{j} E_{0}}{2 \rho r_{0}}\left(2 \frac{\partial^{2} \phi_{j}}{\partial r \partial \eta}-r_{0} \frac{\partial^{3} \phi_{j}}{\partial \eta^{3}}\right)\right\| \\
= & -\frac{1}{2 \rho r_{0}} \frac{\partial^{2} \sigma_{1}}{\partial \eta^{2}} .
\end{aligned}
$$

Initially, the disturbed fluid radius $\delta(\eta, t)$ is independent of the time (also its partial derivative with respect to the time). So, the disturbed fluid radius $\delta(\eta, t)$ has the following initial conditions:

$$
\delta(\eta, 0)=0, \quad \frac{\partial}{\partial t} \delta(\eta, 0)=0
$$

Assuming that the surface tension $\sigma_{1}(\eta)$ has a spatially periodic form, which is independent of the heat and mass transfer. Then, the surface tension function may be written in the following form:

$$
\sigma_{1}(\eta)=-\frac{\Delta \sigma}{2}\left(1+e^{i k \eta}\right)
$$

where $\Delta \sigma$ is a constant variation of the surface tension.

The initial value problem given by (36) and the initial conditions equation (37) can be solved by decomposing the solution into a homogenous and particular parts, $\delta_{h}(\eta, t)$ and $\delta_{p}(\eta, t)$, respectively. Based on the form of the surface tension $\sigma_{1}(\eta)$, which is given in (38), the homogenous solution of the wave function $\delta_{h}(\eta, t)$ may be written in the form

$$
\delta_{h}(\eta, t)=e^{\omega t+i k \eta}
$$

The final form of the general solution of the free surface function, $\delta(\eta, t)=\delta_{h}(\eta, t)+\delta_{p}(\eta, t)$, can be written as follows:

$$
\delta(\eta, t)=-\frac{\Delta \sigma k^{2}}{4 \rho r_{0} b_{0}}\left[\frac{\omega_{-}}{\omega_{+}-\omega_{-}} e^{\omega_{+} t}-\frac{\omega_{+}}{\omega_{+}-\omega_{-}} e^{\omega_{-} t}+1\right] e^{i k \eta}
$$

where, the roots $\omega_{ \pm}$and $b_{0}$ are defined in the appendix.

It is worthwhile to mention here that the final solution in (40) reduces to the same solution obtained earlier by Furlani [3] for the flow of cylindrical microjet surface in the absence of the electric field $\left(E_{0}=0\right)$. It is worthwhile to mention here that the final solution in (40) reduces to the same solution that was obtained earlier by Furlani [3] for the flow of cylindrical microjet surface in the absence of the electric field $\left(E_{0}=0\right)$ with neglecting the porous structure $(\nu=0)$ and with taking the real part only of the free surface function.

\section{Instability of Thermosolutal Marangoni Convection}

If the two-fluid system is a multicomponent system, it is often the case that there may be a preferential concentration of one or more of the components at the interface (e.g., if we consider a system of pure $A$ and pure $B$, which are immiscible, with a third solute component $S$ that is soluble in $A$ and/or $B$ but that is preferentially attracted to the interface), and then the interfacial tension will also be a function of the (surfaceexcess) concentration of these solute components. Both the temperature and the concentrations of adsorbed species can be functions of position on the interface, thus leading to spatial gradients of the surface tension [26]. So, to study the effect of the heat and mass transfer on the interfacial instability of the microcylindrical surface, the surface tension is assumed to be a function of the temperature and the solute concentration and the surface tension will depend on temperature and concentration linearly [29], as follows:

$$
\begin{aligned}
\sigma(\eta, t) & =\sigma_{0}+\sigma_{1}(\eta, t) \\
& =\sigma_{0}\left[1-\gamma_{T}\left(T-T_{0}\right)-\gamma_{C}\left(C-C_{0}\right)\right],
\end{aligned}
$$

where $\gamma_{T}$ and $\gamma_{C}$ denote the temperature and concentration coefficients of the surface tension. Also, $T_{0}$ and $C_{0}$ are the reference temperature and concentration, respectively. Note that (41) is the same as (33) with $\sigma_{1}(z)$ being $\sigma_{1}(\eta, t)$ that depends on the heat and mass transfer as in (41). $\sigma_{0}$ is the unperturbed constant value of the surface tension.

To determine the form of the surface tension, we need firstly to obtain the solution of the heat and concentration equations. Again, the analysis will be based on the normal modes technique as given by

$$
\begin{aligned}
& T(r, \eta, t)=T_{s}(r) e^{\omega t+i k \eta}, \\
& C(r, \eta, t)=C_{s}(r) e^{\omega t+i k \eta} .
\end{aligned}
$$

With the help of the coordinate transformation in (34), the energy and concentration equations (see (5) and (6)) may be written in the following forms:

$$
\begin{aligned}
& \frac{d^{2} T_{s}}{d r^{2}}+\frac{1}{r} \frac{d T_{s}}{d r}-m^{2} T_{s}=0, \\
& \frac{d^{2} C_{s}}{d r^{2}}+\frac{1}{r} \frac{d C_{s}}{d r}-n^{2} C_{s}=0,
\end{aligned}
$$

where $m^{2}=k^{2}+(\omega / \alpha)$ and $n^{2}=k^{2}+(\omega / \beta)$.

If we consider the diffusion of the thermal energy and the concentration in an infinite isolated column of stationary flow of radius $r_{0}$ then there are no temperature and concentration gradients at $r=r_{0}$. So, the temperature and concentration boundary conditions are as follows [3]:

$$
\begin{aligned}
& T_{s}=T_{1}, \quad C_{s}=C_{1}, \quad \text { at } r=R_{1}, \\
& \frac{\partial T_{s}}{\partial r}=0, \quad \frac{\partial C_{s}}{\partial r}=0, \quad \text { at } r=r_{0} .
\end{aligned}
$$


Equations (43) and (44) represent the standard Bessel's differential equation. Therefore, the general solution of the temperature and concentration function may be written as follows:

$$
\begin{aligned}
& T(r, \eta, t) \\
& \quad=\frac{T_{1}\left[K_{0}^{\prime}\left(m r_{0}\right) I_{0}(m r)-I_{0}^{\prime}\left(m r_{0}\right) K_{0}(m r)\right]}{K_{0}^{\prime}\left(m r_{0}\right) I_{0}\left(m R_{1}\right)-K_{0}\left(m R_{1}\right) I_{0}^{\prime}\left(m r_{0}\right)} e^{\omega t+i k \eta}, \\
& C(r, \eta, t) \\
& =\frac{C_{1}\left[K_{0}^{\prime}\left(n r_{0}\right) I_{0}(n r)-I_{0}^{\prime}\left(n r_{0}\right) K_{0}(n r)\right]}{K_{0}^{\prime}\left(n r_{0}\right) I_{0}\left(n R_{1}\right)-K_{0}\left(n R_{1}\right) I_{0}^{\prime}\left(n r_{0}\right)} e^{\omega t+i k \eta} .
\end{aligned}
$$

On combining the following equations:

(1) the general solution of the heat and mass transfer (see (46)),

(2) the surface tension expression (see (41)),

(3) the expression of the free surface function, which may be given based on the normal modes technique as $\delta(\eta, t)=e^{\omega t+i k \eta}$, into the free surface deferential equation given by (36).

We finally get the transcendental dispersion relation of the instability of the interfacial free surface as

$$
f(\omega, k)=\omega^{2}+a_{1}(k) \omega+b_{1}(\omega, k)=0,
$$

where the functions $a_{1}(k)$ and $b_{1}(\omega, k)$ are defined in the appendix.

It is convenient to write the stability criterion in an appropriate dimensionless form. This can be done in a number of ways depending primarily on the choice of the characteristic length, time, temperature, and mass. Consider the following dimensionless forms depending on the characteristic length $=r_{0}$, the characteristic time $=1 / \widehat{\omega}$, the characteristic temperature $=T_{1}$, and the characteristic mass $=\sigma_{0} / \widehat{\omega}^{2}$, where $\widehat{\omega}$ is a characteristic value of $\omega$. The other dimensionless quantities are given by

$$
\begin{gathered}
k=\frac{k^{*}}{r_{0}}, \quad \rho=\rho^{*} \frac{\sigma_{0}}{r_{0}^{3} \widehat{\omega}^{2}}, \quad E_{0}^{2}=E_{0}^{2 *} \frac{\sigma_{0}}{r_{0}}, \\
\mu=\mu^{*} \frac{\sigma_{0}}{\widehat{\omega} r_{0}}, \quad T=T_{1} T^{*}, \quad \gamma_{T}=\frac{\gamma_{T}^{*}}{T_{1}}, \\
\nu=\nu^{*} \frac{\sigma_{0}}{r_{0}^{3} \widehat{\omega}}, \quad r=r^{*} r_{0}, \quad \omega=\widehat{\omega} \omega^{*}, \\
\alpha=\alpha^{*} r_{0}^{2} \widehat{\omega}, \quad \beta=\beta^{*} r_{0}^{2} \widehat{\omega} .
\end{gathered}
$$

The superscript asterisks refer to the dimensionless quantities. From now on, these will be omitted for simplicity. The dispersion relation in (47) and its coefficients $a_{1}(k)$ and $b_{1}(\omega, k)$ may be written as follows:

$$
f(\omega, k)=\omega^{2}+a(k) \omega+b(\omega, k)=0,
$$

where the functions $a(k)$ and $b(\omega, k)$ are defined in the appendix.

The dimensionless transcendental dispersion relation in (49) describes the instability of the interfacial free surface of the microfluid jet. We will discuss the instability criteria numerically and graphically in the next section.

\section{Numerical Estimations}

In the case of the thermosolutal Marangoni convection, the surface tension depends on the heat and mass transfer and the dispersion relation is the transcendental equation (49). The stability criteria of the system may be discussed by getting the values of the growth rate $\omega$ from the roots of the transcendental equation (49). The positive values of $\omega$ indicate a disturbance that grows with time (instability) and the negative values indicate decays with time (stability). So, to study the instability of the microcylindrical surface, we indicate the relation between the growth rate $\omega$ and the wave number $k$ graphically. In all coming curves, the positive values region of $\omega$ indicates the instability region, denoted by letter $U$. Meanwhile, the negative values region of $\omega$ indicates the stability region, denoted by letter $S$.

It is well known that increasing the wave number enhances the stability of the system. As seen in Figure 2, the stability occurs at $k \cong 0.7$, when the intensity of the electric field $E_{0}=1000$. Meanwhile, at $E_{0}=1$ the stability occurs at $k \cong 1$. This means that the stability occurs at less value of $k$ and at the higher value of $E_{0}$, which means that the system is more stable at highly electric field intensity and the electric field has a stabilizing effect. This is an early result obtained by many researchers $[7,20,24,25]$. Also, we can notice that the maximum instability in all figures, except Figure 2, according to the peak of the curve occurs at $k \cong 0.6$. But at high values of the electric field, the peak of the curve approaches the stability region and the maximum instability occurs at $k \cong 0.4$. This implies that existence of the electric field causes a high stability of the interface.

Figure 3 illustrates the electric field and the growth rate relation. It is clear from this figure that the maximum value of the electric field $E_{0}$ is congruent to the zero value of the growth rate $\omega$. The zero value the growth rate $\omega$ corresponds to the steady state of the interface. This result confirms the same result of Figure 2; that is, the electric field has a stabilizing effect. Figure 4 illustrates the effect of the dielectric constants difference $\widehat{\varepsilon}=\varepsilon_{1}-\varepsilon_{2}$ on the stability picture. The dielectric difference $\widehat{\varepsilon}$ has a stabilizing effect, where the higher value of $\widehat{\varepsilon}$ is identical to the minimum peak value of the curve.

Figures 5-7 illustrate the effect of Darcy's coefficient, the viscosity, and the density of fluid on the stability behavior. The of Darcy's coefficient, the viscosity, and the density of fluid have stabilizing effect for $k \leq 1$ according to reducing the peak of the curve and decreasing the number of the points in the instability region. Figure 5 illustrates the effect of the existence of the porous structure, according to the different values of the Darcy's coefficient, on the stability picture. It is clear that the microcylindrical surface is more stable, in case of the flow through a porous medium for majority values of the wave number, due to an increase in the values of the 


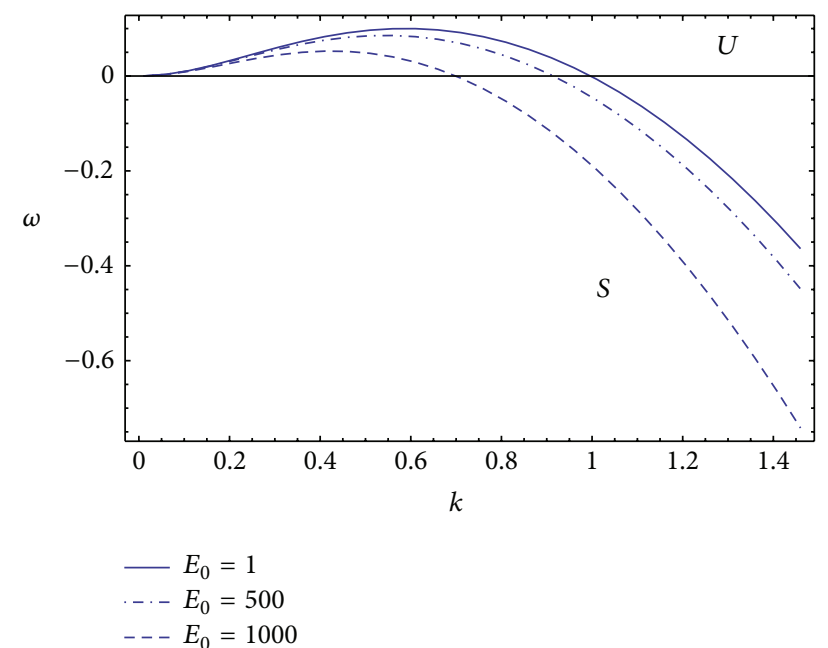

FIGURE 2: Stability diagram for a system having the particulars $\rho=1$, $\mu=0.5, R_{1}=10^{-5}, R_{2}=10, v=0.5, \varepsilon_{1}=5 \times 10^{-5}, \varepsilon_{2}=10^{-4}$, $r_{0}=5 \times 10^{-3}, \gamma_{T}=0.01, \gamma_{C}=0.2, \alpha=0.01, T_{1}=0.05, C_{1}=0.03$, and $\beta=0.2$.

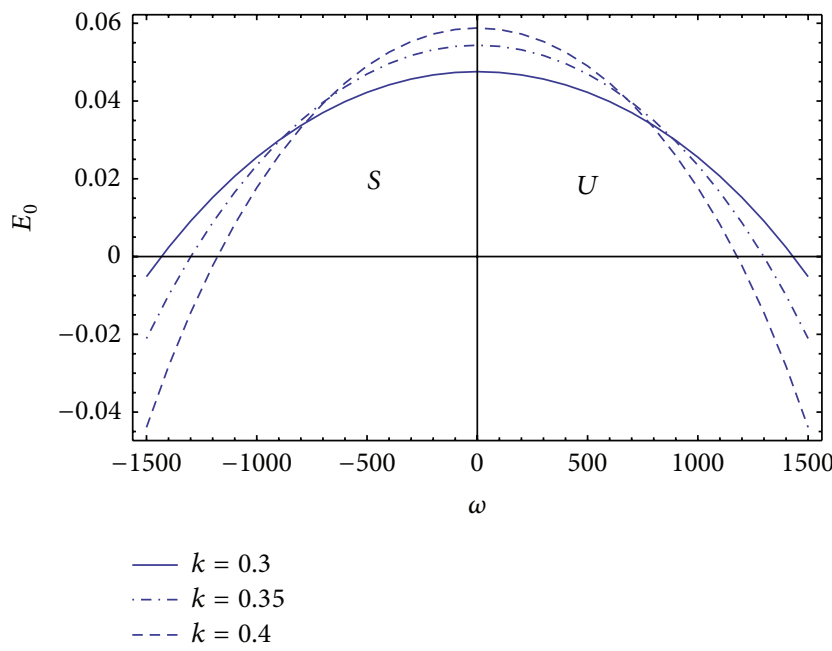

Figure 3: The electric field plotted versus the growth rate at $\rho=1$, $\mu=0.5, R_{1}=10^{-5}, R_{2}=10, E_{0}=10, \gamma_{T}=0.01, \gamma_{C}=0.03$, $r_{0}=5 \times 10^{-3}, \alpha=0.01, C_{1}=10, T_{1}=10, \beta=0.2, \varepsilon_{1}=5 \times 10^{-5}$, and $\varepsilon_{2}=10^{-4}$.

Darcy's coefficients in comparison with the case of the pure flow $(v=0)$. This is due to the fact that the greatest peak value of the curve in the instability region is at $\nu=0$. Hence the Darcy's coefficient $\nu$ is equal to $\mu / \lambda$, where $\lambda$ is the permeability of the porous medium, so an increase in the values of the Darcy's coefficients corresponds to a decrease in the permeability of the porous medium. This in turn restricts the streaming velocity of the fluid flow. It is known that when the streaming velocity decreases, the stability of the system increases. In other words, when the permeability of the medium increases ( $\lambda$ increases and $v$ decreases) the holes of the porous medium are very large and the resistance of the medium may be neglected so that the streaming velocity

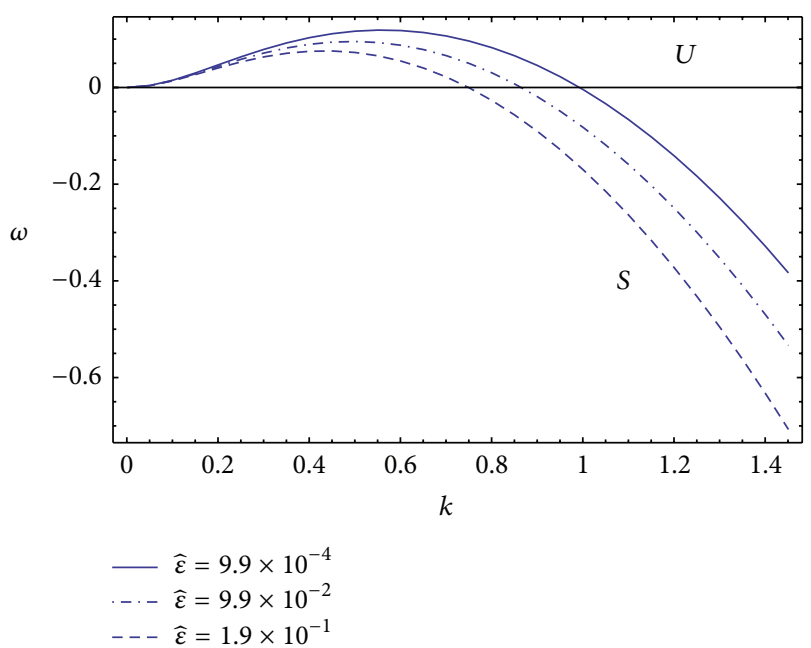

FIGURE 4: Stability diagram for a system having the particulars $\rho=1$, $\mu=0.5, R_{1}=10^{-5}, R_{2}=10, E_{0}=10, \gamma_{T}=0.01, \gamma_{C}=0.03$, $r_{0}=5 \times 10^{-3}, \alpha=0.01, C_{1}=0.04, T_{1}=1$, and $\beta=0.2$.

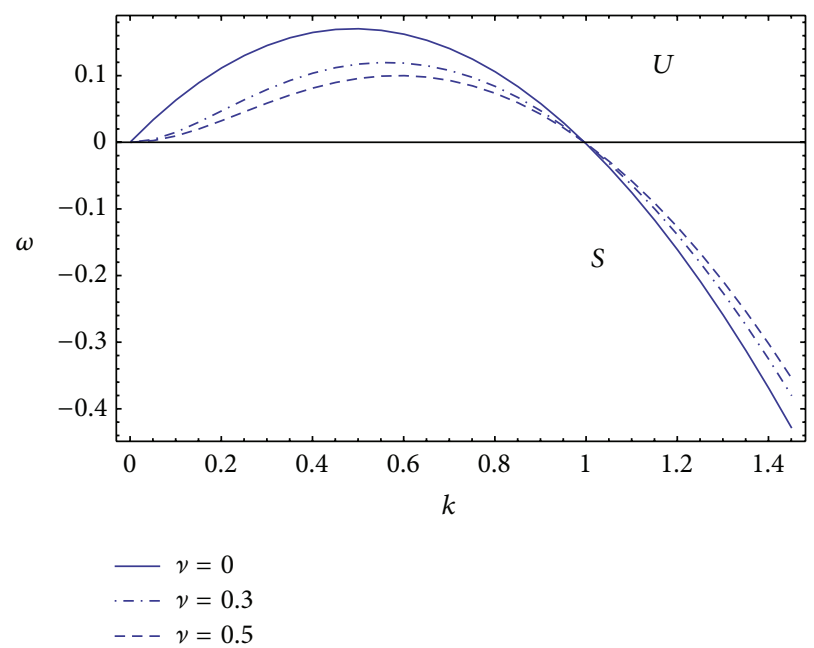

FIGURE 5: Stability diagram for a system having the particulars $\rho=1$, $\mu=0.5, R_{1}=10^{-5}, R_{2}=10, E_{0}=10, \varepsilon_{1}=5 \times 10^{-5}, \varepsilon_{2}=10^{-4}$, $r_{0}=5 \times 10^{-3}, \gamma_{T}=0.01, \gamma_{C}=0.2, \alpha=0.01, T_{1}=0.05, C_{1}=0.03$, and $\beta=0.2$.

increases and causes instability of the system. The same result was illustrated experimentally by Catton and Chung [21], where the authors studied the interfacial stability between the water and the steam. Two porous media were studied experimentally: a bead of glass with diameter $0.6,2$, and 6 and with permeability $0.14,1.57$, and $18.0\left(\times 10^{-9} \mathrm{~m}^{2}\right)$, respectively, and a bead of steel with diameter 4 and with permeability 9.02 $\left(\times 10^{-9} \mathrm{~m}^{2}\right)$. It was found that in the more permeable cylinder the steam bubble is chaotic just after it has been formed. This means that the interface becomes unstable and the chaotic turbulence becomes more and more profound as the water flow rate increases (according to increasing the permeability of the porous medium). 


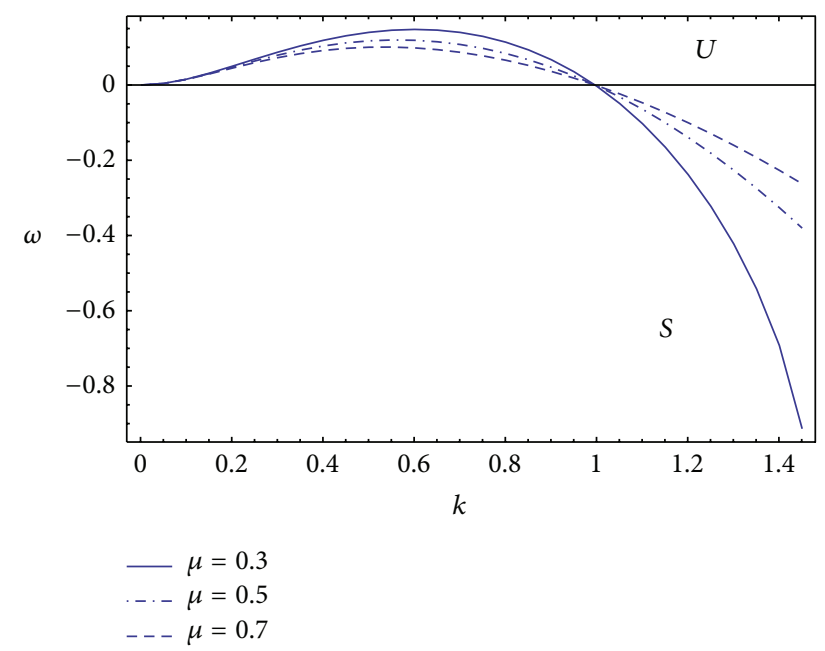

FIGURE 6: Stability diagram for a system having the particulars $\rho=1$, $\nu=0.3, R_{1}=10^{-5}, R_{2}=10, E_{0}=10, \varepsilon_{1}=5 \times 10^{-5}, \varepsilon_{2}=10^{-4}$, $r_{0}=5 \times 10^{-3}, \gamma_{T}=0.01, \gamma_{C}=0.2, \alpha=0.01, T_{1}=0.05, C_{1}=0.03$, and $\beta=0.2$.

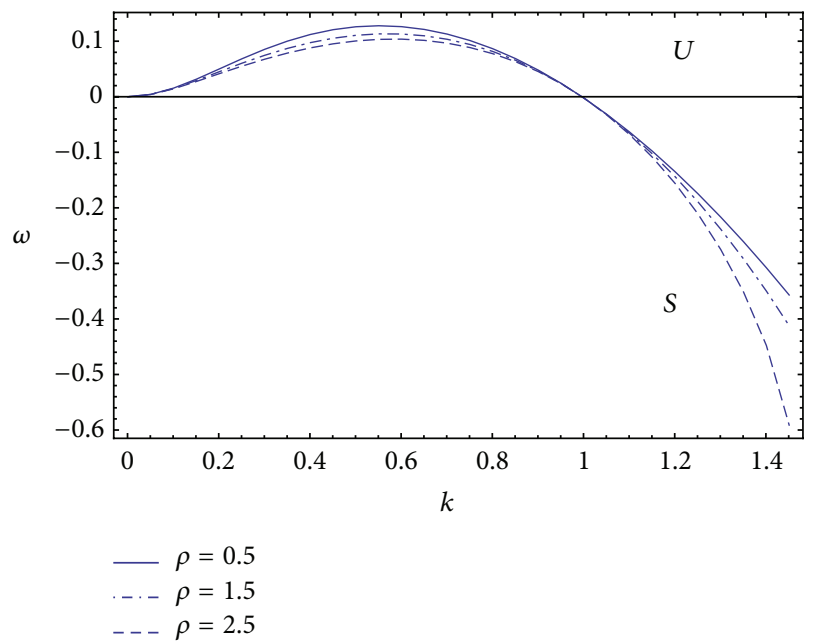

FIGURE 7: Stability diagram for a system having the particulars $v=$ $0.3, \mu=0.5, R_{1}=10^{-5}, R_{2}=10, E_{0}=10, \varepsilon_{1}=5 \times 10^{-5}, \varepsilon_{2}=10^{-4}$, $r_{0}=5 \times 10^{-3}, \gamma_{T}=0.01, \gamma_{C}=0.2, \alpha=0.01, T_{1}=0.05, C_{1}=0.03$, and $\beta=0.2$.

The viscosity $\mu$ and the density $\rho$ have a stabilizing effect, for larger values of the wave number, as shown in Figures 6 and 7. This influence occurs due to an increase in the viscosity damping and in the inertia. So, the interface of the microcylindrical surface becomes stable with an increase in the viscosity and the density.

The effect of heat transfer on the free surface is illustrated in Figure 8. To study the instability influence, according to the heat transfer, we notice that the temperature coefficient is $\gamma_{T}= \pm\left(1 / \sigma_{0}\right)(d \sigma / d T)[30]$, where $\gamma_{T}$ has a positive or negative sign according to the increasing or decreasing of the surface tension. When $T<T_{1}$, the axial microsolid cylinder, which induces the heat and mass gradients, is heated and then

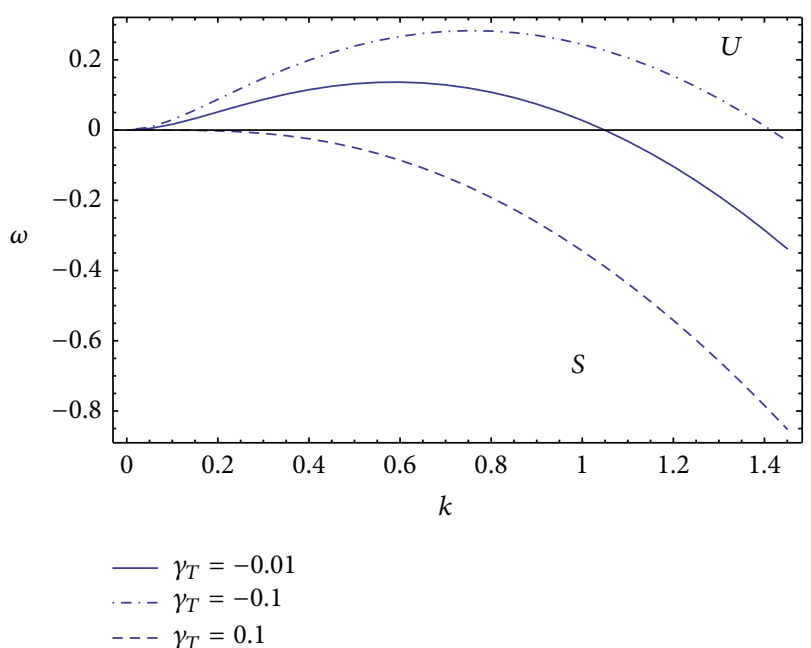

FIGURE 8: Stability diagram for a system having the particulars $\rho=1$, $\mu=0.5, R_{1}=10^{-5}, R_{2}=10, E_{0}=10, \varepsilon_{1}=5 \times 10^{-5}, \varepsilon_{2}=10^{-4}$, $r_{0}=5 \times 10^{-3}, \gamma_{C}=-0.03, \alpha=0.01, \nu=0.3, C_{1}=0.03, \beta=0.2$, and $T_{1}=10$.

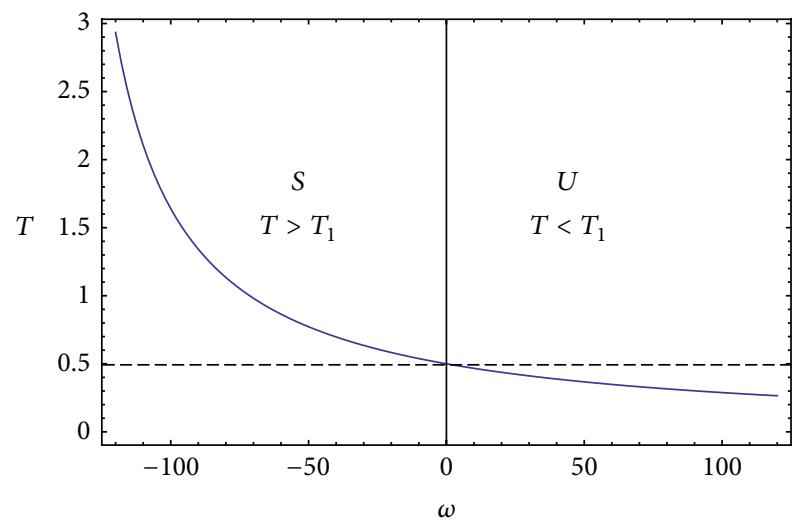

FIGURE 9: The temperature plotted versus the growth rate at $\rho=1$, $\mu=0.5, R_{1}=10^{-5}, R_{2}=10, E_{0}=10, \gamma_{T}=0.01, \gamma_{C}=0.03$, $r_{0}=5 \times 10^{-3}, \alpha=0.01, C_{1}=10, T_{1}=0.5, \beta=0.2, \varepsilon_{1}=5 \times 10^{-5}$, and $\varepsilon_{2}=10^{-4}$.

the temperate transfers from it to the free surface by convection currents. This in turn tends to evaporate the fluid easily and provokes higher instabilities at the interface. So, in this case, $\gamma_{T}$ has a negative value according to the decreasing of the surface tension. Conversely, $\gamma_{T}$ has a positive value when $T>$ $T_{1}$. In this case the free surface loses its temperature that is transferred to the core region of the flow jet and hence the free surface becomes stable. This phenomenon was illustrated in Figure 8 where the temperature coefficient $\gamma_{T}$ has a stabilizing effect when it is positive and the inverse occurs at $\gamma_{T}<0$. In other words, heating the axial microsolid surface $T_{1}$ has destabilizing effect at the interface and conversely for cooling. Figure 9 confirms this result. We notice from this figure that the unstable state, for the positive values of the growth rate $\omega$, occurs at the temperature values corresponding to $T<$ $T_{1}$. Meanwhile, the temperature $T>T_{1}$ is for the negative 


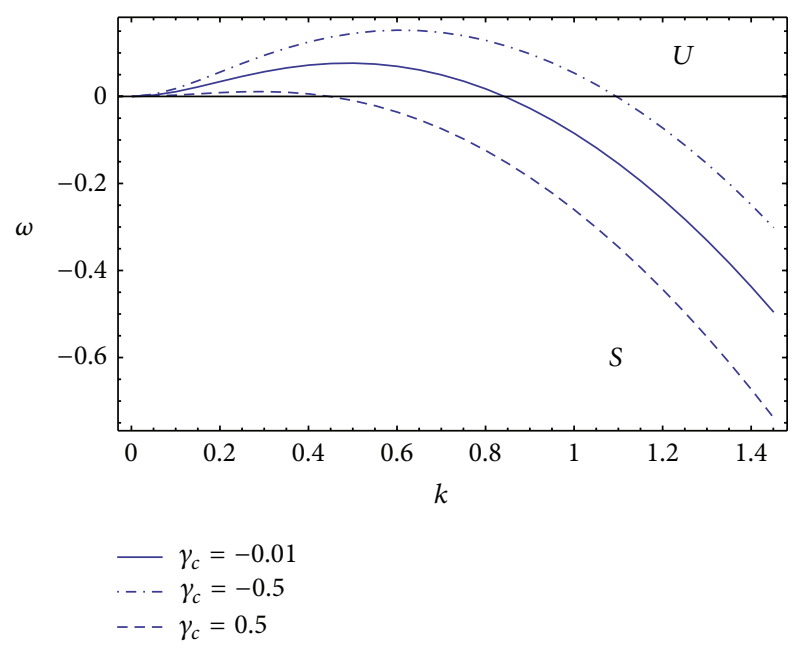

FIGURE 10: Stability diagram for a system having the particulars $\rho=$ $1, \mu=0.5, R_{1}=10^{-5}, R_{2}=10, E_{0}=10, \varepsilon_{1}=5 \times 10^{-5}, \varepsilon_{2}=10^{-4}$, $r_{0}=5 \times 10^{-3}, \gamma_{T}=-0.01, \alpha=0.01, \nu=0.3, T_{1}=1, \beta=0.2$, and $C_{1}=10$.

growth rate in the stability region. This result was obtained experimentally by D'Aubeterre et al. [31]. The authors stated that the temperature has a destabilizing effect due to the fact that a substance is more volatile for increasing the temperate; this means that when a temperature gradient occurs, the substance tends to evaporate easily and provokes higher instabilities at the interface. Also, depending on the alcohol studied [31], turbulence begins at the interface at different temperature gradients. Instabilities begin near the interface, showing a sinuous movement. At higher temperatures and temperature gradients, instabilities increase and movements become continuous. The most evident effect occurs when temperature gradients are $15-17^{\circ} \mathrm{C}$; at these values a sinuous wave was observed [31].

Similar behavior can be noticed for the concentration as seen in Figure 10. In this figure we use $\gamma_{C}= \pm\left(1 / \sigma_{0}\right)(d \sigma / d C)$ [30]. The concentration coefficients of the surface tension $\gamma_{C}$ become negative if $C<C_{1}$. This implies that the concentration at the interface is less than the concentration at the core region. Because the surface tension is proportional with the concentration, the surface tension at the free surface accordingly reduces. So, the free surface loses its interfacial rigidity and its ability to movement increases. Therefore the instability of the free surface occurs. On the other hand, when the concentration of the interface is higher than that of the core region, the stability in the interface occurs. This means an increase of the interfacial rigidity, which in turn restricts any surface movements or Marangoni convection. Therefore, the surface becomes stable when $\gamma_{C}>0$ and the instability occurs for $\gamma_{C}<0$, as shown in Figure 10. Figure 11 confirms this result, where the concentration is plotted versus the growth rate. The region of the positive values of the growth rate $\omega$, that is, the unstable region, occurs at $C<C_{1}$. Meanwhile, the stable region, that is, the negative growth rate values, occurs at $C>C_{1}$. Similar results were obtained by Agble and Mendes [32] and compared with experimental results.

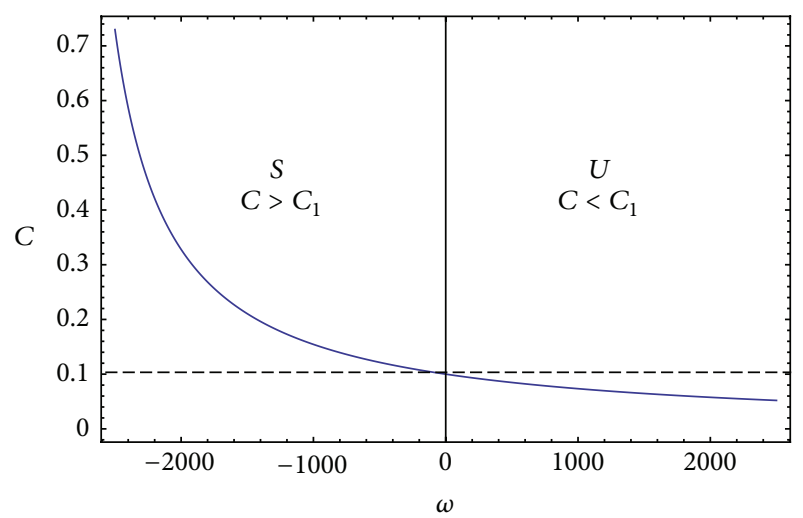

FIGURE 11: The concentration plotted versus the growth rate at $\rho=1$, $\mu=0.5, R_{1}=10^{-5}, R_{2}=10, E_{0}=10, \gamma_{T}=0.01, \gamma_{C}=0.03$, $r_{0}=5 \times 10^{-3}, \alpha=0.01, C_{1}=0.1, T_{1}=0.5, \beta=0.2, \varepsilon_{1}=5 \times 10^{-5}$, and $\varepsilon_{2}=10^{-4}$.

TABLE 1: The root of the growth rate $\omega$ for different values of the micro radius $r_{0}$ at $\rho=1, \mu=0.5, \varepsilon_{1}=10^{-5}, \varepsilon_{2}=10^{-3}, R_{1}=10^{-5}$, $E_{0}=10, R_{2}=10, \gamma_{T}=0.5, \gamma_{C}=0.03, \alpha=0.01, \beta=0.2, T_{1}=1$, $C_{1}=0.04$, and $v=0.3$

\begin{tabular}{lcc}
\hline$r_{0}$ & $k$ & $\omega$ \\
\hline \multirow{3}{*}{$5 \times 10^{-3}$} & $10^{-3}$ & $1.63164 \times 10^{-6}$ \\
& $10^{-1}$ & 0.0148314 \\
& $10^{1}$ & -48.76930 \\
& $10^{2}$ & -4998.710 \\
\hline \multirow{2}{*}{$5 \times 10^{-1}$} & $10^{-3}$ & $1.02608 \times 10^{-8}$ \\
& $10^{-1}$ & 0.0146367 \\
& $10^{1}$ & -48.82320 \\
& $10^{2}$ & -4998.800 \\
\hline
\end{tabular}

Finally, the instability criterion of the system can be discussed by studying the roots of the dispersion relation (49) to get the values of the growth rate $\omega$, as shown in Table 1 . The negative values of the roots of $\omega$ indicate stability of the microcylindrical surface and the inverse occurs of the positive root values. It can be noticed from the roots of the growth rate $\omega$ that the interface becomes stable when the microradius $r_{0}$ increases which means an increase in the inertia with $r_{0}$.

\section{Conclusion}

In this paper, we have examined the influence of the existence of the porous structure on the EHD instability of the free microcylindrical fluid surface with thermosolutal Marangoni convection. The surface tension depends linearly on the heat and mass transfer. The main results of our study can be epitomized in the following points.

(1) The existence of the porous structure has a stabilizing effect for the free surface. Existence of the porous medium restricts the streaming velocity of the fluid. Similar behavior was obtained experimentally by [21] for the steam-liquid interface thorough a porous structure. 
(2) As it is known, the electric field has a stabilizing effect, and this result coincides with the previously published results in the literature $[7,20,24,25]$.

(3) The heating of the axial microsolid cylinder reduces the surface tension and makes the free surface volatile easily and hence the surface becomes unstable and the inverse occurs for cooling the axial microsolid cylinder. The same results were obtained experimentally by [31].

(4) The surface tension is proportional to the concentration variation. So, high concentration at the free surface inhibits the movement of the surface. This means stability of the free surface. The inverse behavior was obtained for a high concentration at the core region. Similar trend was obtained experimentally by [32].

Finally, our calculation of the free surface function was compared with the previous results of [3] in the absence of the porous medium and the electric field. Also, the present results were compared with the experimental results of [21, 31, 32]. There was compatibility between the results.

\section{Appendix}

(1) The functions $g_{1}(k), g_{2}(k), G_{1}(k)$, and $G_{2}(k)$ are given as

$$
\begin{aligned}
& g_{1}(k)=K_{0}\left(k r_{0}\right) I_{0}^{\prime}\left(k R_{1}\right)-I_{0}\left(k r_{0}\right) K_{0}^{\prime}\left(k R_{1}\right), \\
& g_{2}(k)=K_{0}\left(k r_{0}\right) I_{0}^{\prime}\left(k R_{2}\right)-I_{0}\left(k r_{0}\right) K_{0}^{\prime}\left(k R_{2}\right), \\
& G_{1}(k)=I_{0}^{\prime}\left(k r_{0}\right) K_{0}^{\prime}\left(k R_{1}\right)-K_{0}^{\prime}\left(k r_{0}\right) I_{0}^{\prime}\left(k R_{1}\right), \\
& G_{2}(k)=I_{0}^{\prime}\left(k r_{0}\right) K_{0}^{\prime}\left(k R_{2}\right)-K_{0}^{\prime}\left(k r_{0}\right) I_{0}^{\prime}\left(k R_{2}\right) .
\end{aligned}
$$

(2) The roots $\omega_{ \pm}$are defined as

$$
\omega_{ \pm}=\frac{-a_{0} \pm \sqrt{a_{0}^{2}-4 b_{0}}}{2},
$$

where

$$
\begin{gathered}
a_{0}=\frac{3 \mu}{\rho} k^{2}+\frac{\nu}{\rho} \\
b_{0}=-\frac{\sigma_{0} k^{2}}{2 \rho r_{0}}+\frac{\sigma_{0} r_{0} k^{4}}{2 \rho} \\
+\frac{E_{0}^{2} k^{3} r_{0}}{2 \rho} \frac{\left(\varepsilon_{2}-\varepsilon_{1}\right)^{2} g_{1}(k) g_{2}(k)}{\varepsilon_{2} G_{2}(k) g_{1}(k)-\varepsilon_{1} G_{1}(k) g_{2}(k)} .
\end{gathered}
$$

(3) The functions $a_{1}(k)$ and $b_{1}(\omega, k)$ are defined as

$$
\begin{aligned}
a_{1}(k)= & \frac{3 \mu}{\rho} k^{2}+\frac{\nu}{\rho}, \\
b_{1}(\omega, k)= & -\frac{\sigma_{0} k^{2}}{2 \rho r_{0}}+\frac{\sigma_{0} r_{0} k^{4}}{2 \rho} \\
& +\frac{E_{0}^{2} k^{3} r_{0}}{2 \rho} \frac{\left(\varepsilon_{2}-\varepsilon_{1}\right)^{2} g_{1}(k) g_{2}(k)}{\varepsilon_{2} G_{2}(k) g_{1}(k)-\varepsilon_{1} G_{1}(k) g_{2}(k)} \\
& +\frac{\sigma_{0} k^{2}}{2 \rho r_{0}}\left[\gamma_{T} T_{s}\left(r_{0}\right)+\gamma_{C} C_{s}\left(r_{0}\right)\right] .
\end{aligned}
$$

Also, the functions $a(k)$ and $b(\omega, k)$ are defined as

$$
\begin{gathered}
a(k)=\frac{3 \mu}{\rho} k^{2}+\frac{\nu}{\rho}, \\
b(\omega, k)=\frac{1}{2 \rho}\left(-k^{2}+k^{4}\right) \\
+\frac{E_{0}^{2} k^{3}}{2 \rho} \frac{\left(\varepsilon_{2}-\varepsilon_{1}\right)^{2} g_{1}(k) g_{2}(k)}{\varepsilon_{2} G_{2}(k) g_{1}(k)-\varepsilon_{1} G_{1}(k) g_{2}(k)} \\
+\frac{k^{2}}{2 \rho}\left[\gamma_{T} T_{s}\left(r_{0}\right)+\gamma_{C} C_{s}\left(r_{0}\right)\right] .
\end{gathered}
$$

\section{Nomenclature}

$\begin{array}{ll}C: & \text { Concentration } \\ C_{0}: & \text { Concentration at the interface } \\ C_{1}: & \text { Concentration at the microcylindrical } \\ E_{0}: & \text { solid axis } \\ F: & \text { Intensity of the electric field } \\ h: & \text { Interface function } \\ H: & \text { Radius for the disturbed fluid surface } \\ I_{0}, K_{0}: & \text { Curvature of the interface } \\ k: & \text { Modified Bessel's function of the first } \\ \widehat{n}: & \text { Wave number } \\ P: & \text { Unit normal vector to the interface } \\ P_{0}(z, t), P_{2}(z, t): & \text { Perturbed pressure functions } \\ (r, \theta, z): & \text { Cylindrical coordinates } \\ r_{0}: & \text { Microradius of the fluid cylindrical } \\ R_{1}: & \text { surface } \\ R_{2}: & \text { Microradius of the cylindrical solid axis } \\ t: & \text { Radius of the surrounded vacuum } \\ \widehat{t}: & \text { Time } \\ T: & \text { Unit tangential vector to the interface } \\ T_{0}: & \text { Temperature } \\ T_{1}: & \text { Temperature at the interface } \\ u(z, t): & \text { Temperature at the surface of } \\ u_{0}: & \text { microcylindrical axis } \\ v: & \text { Axial streaming velocity function } \\ & \text { Constant streaming velocity } \\ & \text { Velocity vector }\end{array}$


$v_{r}, v_{z}: \quad$ Radial and axial velocity components

$v_{0}(z, t), v_{2}(z, t)$ : Perturbed velocity functions.

\section{Greek Symbols}

$\begin{array}{ll}\alpha: & \text { Thermal diffusivity } \\ \beta: & \text { Mass diffusivity } \\ \sigma(z, t): & \text { Surface tension } \\ \sigma_{0}: & \text { Unperturbed surface tension } \\ \sigma_{1}(z, t): & \text { Perturbed surface tension } \\ \Delta \sigma: & \text { A constant variation of the surface } \\ & \text { tension } \\ \gamma_{T}= \pm\left(1 / \sigma_{0}\right)(d \sigma / d T): & \text { Temperature coefficient of the } \\ & \text { surface tension } \\ \gamma_{C}= \pm\left(1 / \sigma_{0}\right)(d \sigma / d C): & \text { Concentration coefficient of the } \\ \phi: & \text { surface tension } \\ \tau_{i j}: & \text { Electric potential function } \\ \omega: & \text { Stress tensor } \\ \mu: & \text { Growth rate of the surface wave } \\ \mu_{\mathrm{eff}}: & \text { Viscosity } \\ \nu: & \text { Effective viscosity } \\ \varepsilon_{1}, \varepsilon_{2}: & \text { Darcy's coefficient } \\ \delta(z, t): & \text { Dielectric constants of the liquid } \\ \eta=z-v_{0} t: & \text { and vacuum, respectively } \\ \rho: & \text { Dimensionless radius of the } \\ & \text { disturbed fluid surface } \\ & \text { Transformation of the axial } \\ & \text { distance } \\ & \text { Density. } \\ & \end{array}$

\section{References}

[1] S. Bhattacharyya and A. K. Singh, "Augmentation of heat transfer from a solid cylinder wrapped with a porous layer," International Journal of Heat and Mass Transfer, vol. 52, no. 7-8, pp. 1991-2001, 2009.

[2] R. E. S. Moya, A. T. Prata, and J. A. B. C. Neto, "Experimental analysis of unsteady heat and moisture transfer around a heated cylinder buried into a porous medium," International Journal of Heat and Mass Transfer, vol. 42, no. 12, pp. 2187-2198, 1999.

[3] E. P. Furlani, "Temporal instability of viscous liquid microjets with spatially varying surface tension," Journal of Physics A, vol. 38, no. 1, pp. 263-276, 2005.

[4] E. P. Furlani and M. S. Hanchak, "Nonlinear analysis of the deformation and breakup of viscous microjets using the method of lines," International Journal for Numerical Methods in Fluids, vol. 65 , no. 5 , pp. 563-577, 2011.

[5] Z. Gao, "Instability of non-Newtonian jets with a surface tension gradient," Journal of Physics A, vol. 42, no. 6, Article ID 065501, 2009.

[6] Z. Gao and K. Ng, "Temporal analysis of power law liquid jets," Computers \& Fluids, vol. 39, no. 5, pp. 820-828, 2010.

[7] A. A. Mohamed and N. K. Nayyar, "Electrohydrodynamic stability of a liquid jet," Journal of Physics A, vol. 3, no. 3, pp. 296303, 1970.

[8] C. H. Chen, "Marangoni effects on forced convection of powerlaw liquids in a thin film over a stretching surface," Physics Letters A, vol. 370, no. 1, pp. 51-57, 2007.

[9] L. G. Napolitano, "Microgravity fluid dynamics," in Proceedings of the 2nd Levitch Conference, Washington, DC, USA, 1978.
[10] L. G. Napolitano, "Marangoni boundary layers," in Proceedings of the 3rd European Symposium on Material Science in Space, ESA SP-142, Grenoble, France, June 1979.

[11] S. Hansen, G. W. M. Peters, and H. E. H. Meijer, "The effect of surfactant on the stability of a fluid filament embedded in a viscous fluid," Journal of Fluid Mechanics, vol. 382, pp. 331-349, 1999.

[12] M. E. Timmermans and J. R. Lister, "The effect of surfactant on the stability of a liquid thread," Journal of Fluid Mechanics, vol. 459, pp. 289-306, 2002.

[13] S. Kwak, M. M. Fyrillas, and C. Pozrikidis, "Effect of surfactants on the instability of a liquid thread. Part II: Extensional flow," International Journal of Multiphase Flow, vol. 27, no. 1, pp. 3960, 2001.

[14] R. V. Craster, O. K. Matar, and D. T. Papageorgiou, "Pinchoff and satellite formation in surfactant covered viscous threads," Physics of Fluids, vol. 14, no. 4, pp. 1364-1376, 2002.

[15] B. Ambravaneswaran and O. A. Basaran, "Effects of insoluble surfactants on the nonlinear deformation and breakup of stretching liquid bridges," Physics of Fluids, vol. 11, no. 5, pp. 9971015, 1999.

[16] P. T. McGough and O. A. Basaran, "Repeated formation of fluid threads in breakup of a surfactant-covered jet," Physical Review Letters, vol. 96, no. 5, Article ID 054502, 4 pages, 2006.

[17] D. M. Christopher and B. Wang, "Prandtl number effects for Marangoni convection over a flat surface," International Journal of Thermal Sciences, vol. 40, no. 6, pp. 564-570, 2001.

[18] J. Straub, "The role of surface tension for two-phase heat and mass transfer in the absence of gravity," Experimental Thermal and Fluid Science, vol. 9, no. 3, pp. 253-273, 1994.

[19] K. R. Rajagopal, "On a hierarchy of approximate models for flows of incompressible fluids through porous solids," Mathematical Models and Methods in Applied Sciences, vol. 17, no. 2, pp. 215-252, 2007.

[20] A. E. K. Elcoot and G. M. Moatimid, "Nonlinear instability of finitely conducting cylindrical flows through porous media," Physica A, vol. 343, no. 1-4, pp. 15-35, 2004.

[21] I. Catton and M. Chung, "An experimental study of steam injection into a uniform water flow through porous media," Wärme-und Stoffübertragung, vol. 27, no. 1, pp. 29-35, 1992.

[22] D. A. Nield and A. Bejan, Convection in Porous Media, Springer, New York, NY, USA, 3rd edition, 2006.

[23] S. Liu and J. H. Masliyah, "Dispersion in porous media," in Handbook of Porous Media, Taylor \& Francis, Boca Raton, Fla, USA, 2nd edition, 2005.

[24] J. R. Melcher, Field Coupled Surface Waves, MIT Press, Cambridge, Mass, USA, 1963.

[25] J. R. Melcher, Continuum Electrodynamics, MIT Press, Cambridge, Mass, USA, 1981.

[26] L. G. Leal, Advanced Transport Phenomena, Cambridge Series in Chemical Engineering, Cambridge University Press, Cambridge, UK, 2007.

[27] G. M. Moatimid, "Nonlinear instability of two dielectric viscoelastic fluids," Canadian Journal of Physics, vol. 82, no. 12, pp. 1109-1133, 2004.

[28] H. H. Woodson and J. R. Melcher, Electromechanical Dynamics Part II: Fields, Forces, and Motion, John Wiley \& Sons, New York, NY, USA, 1986.

[29] E. Magyari and A. J. Chamkha, "Exact analytical results for the thermosolutal MHD Marangoni boundary layers," International Journal of Thermal Sciences, vol. 47, no. 7, pp. 848-857, 2008. 
[30] S. Slavtchev, P. Kalitzova-Kurteva, and M. A. Mendes, "Marangoni instability of liquid-liquid systems with a surface-active solute," Colloids and Surfaces A, vol. 282-283, pp. 37-49, 2006.

[31] A. D’Aubeterre, R. da Silva, and M. E. Aguilera, "Experimental study on Marangoni effect induced by heat and mass transfer," International Communications in Heat and Mass Transfer, vol. 32, no. 5, pp. 677-684, 2005.

[32] D. Agble and M. A. Mendes-Tatsis, "Prediction of Marangoni convection in binary liquid-liquid systems with added surfactants," International Journal of Heat and Mass Transfer, vol. 44, no. 7, pp. 1439-1449, 2001. 


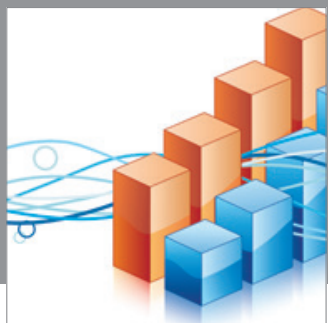

Advances in

Operations Research

mansans

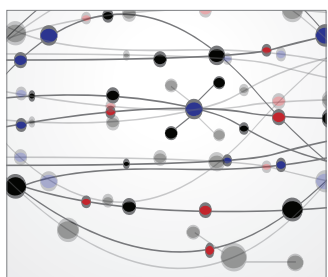

The Scientific World Journal
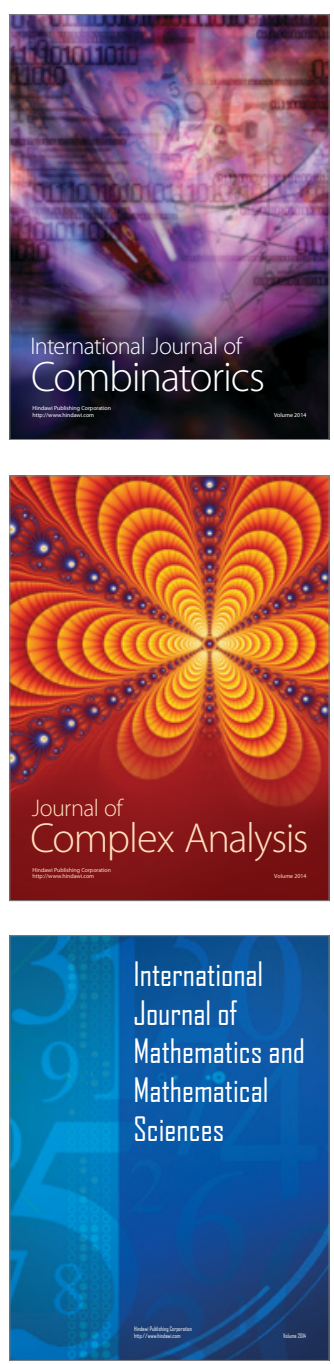
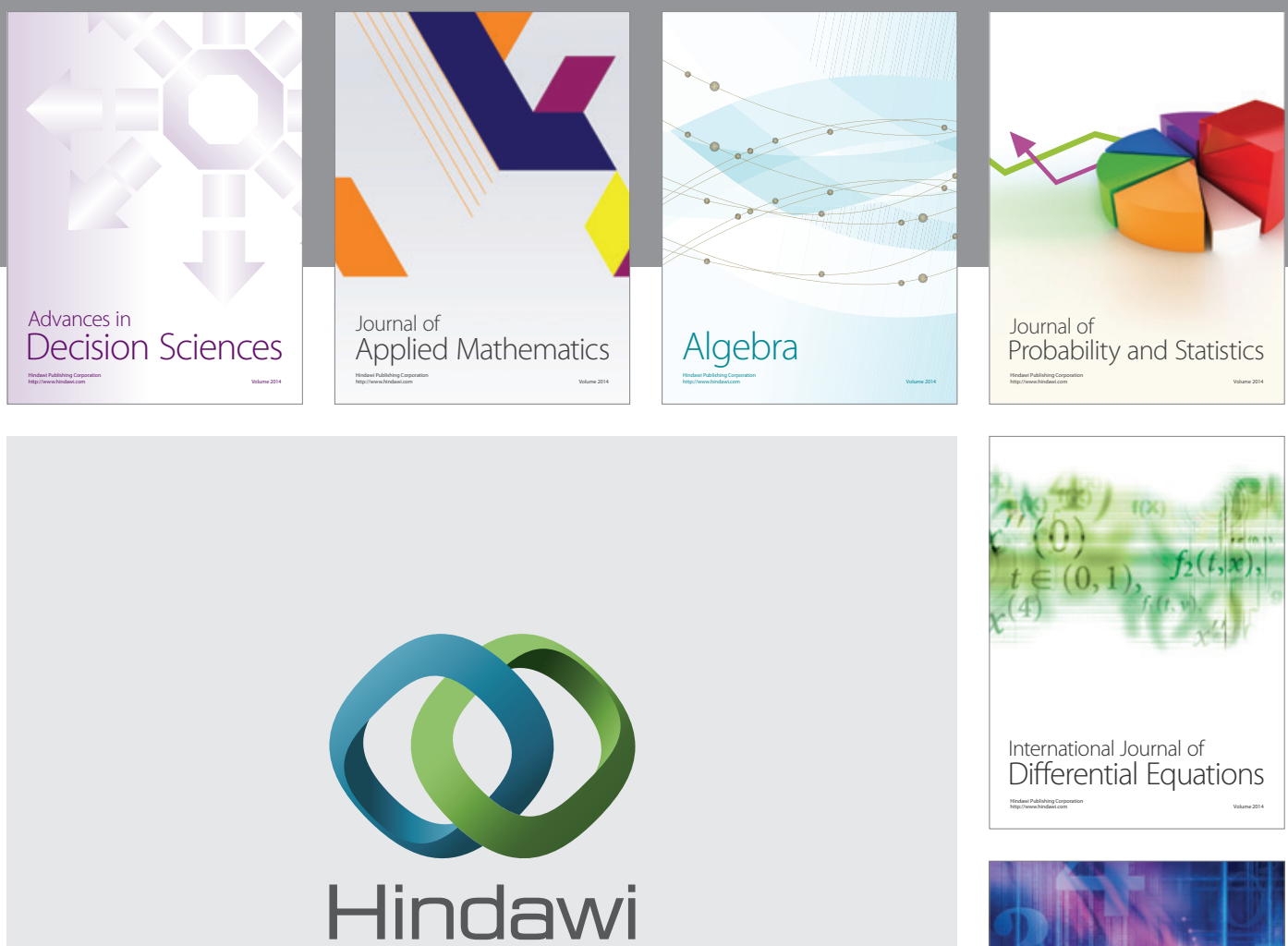

Submit your manuscripts at http://www.hindawi.com
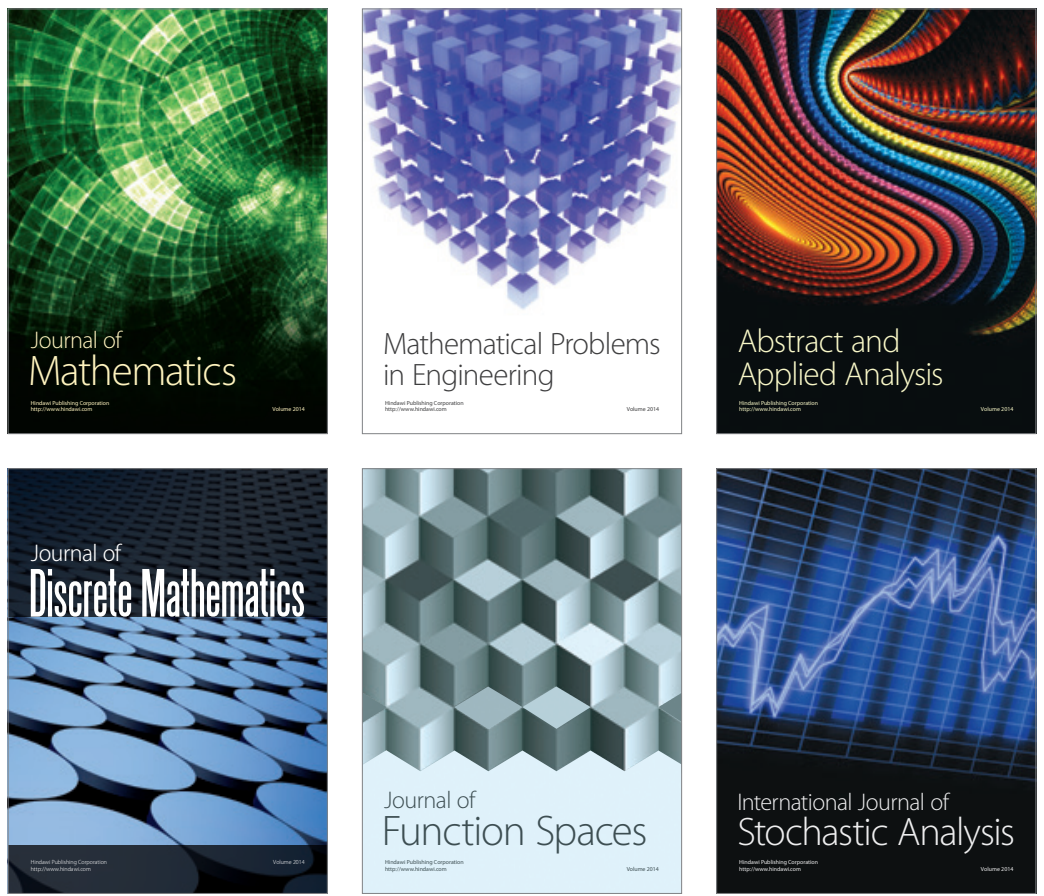

Journal of

Function Spaces

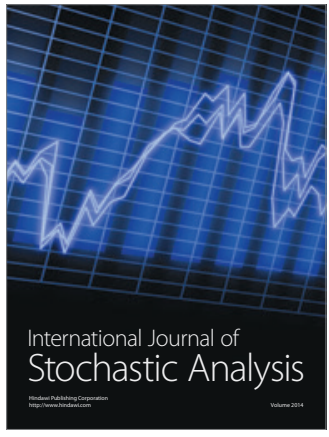

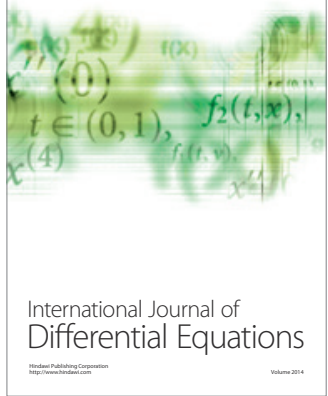
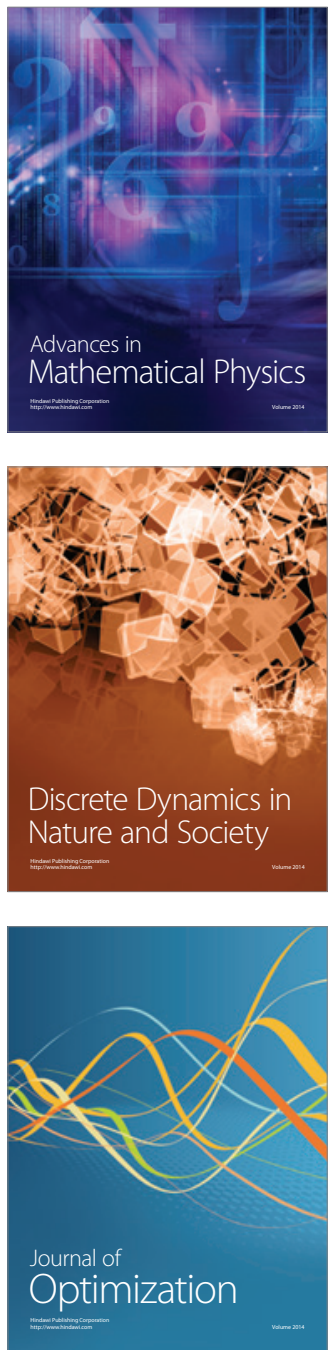